\title{
High Voltage Crowbar Protection for the Large CDF Axial Drift Chamber
}

\author{
M. Binkley, A. Mukherjee, W.Stuermer, and R. L. Wagner
}

\begin{abstract}
The Central Outer Tracker (COT) is a big cylindrical drift chamber that provides charged particle tracking for the Collider Detector at Fermilab experiment. To protect the COT, the large stored energy in the high voltage system needs to be removed quickly when a problem is sensed. For the high voltage switch, a special-order silicon-controlled-rectifier was chosen over more readily available integrated gate bipolar transistors because of layout and reliability questions. The considerations concerning the high voltage switch, the prototype performance, and the experience of more than two years of running are described.
\end{abstract}

\section{INTRODUCTION}

The Collider Detector at Fermilab (CDF)[1] is a large magnetic detector built to study $2 \mathrm{TeV}$ PbarP collisions at the Fermilab Tevatron Collider. At its center is the Central Outer Tracker (COT)[2], a large cylindrical tracking chamber coaxial with the beam that measures the 3-momentum of charged particles with excellent resolution.

With more than 30,000 sense wires at high voltage, the COT operates with a large stored electrical energy. For practical reasons the high voltage is distributed using daisy chains to many wires in parallel, each with almost $1 \mathrm{nF}$ capacitance to ground. When an over-current condition is sensed, it is necessary to quickly remove the stored energy to prevent a problem wire from being recharged from its neighboring wires and undergoing multiple discharges.

Section II reviews the mechanical properties of the chamber. Section III describes the electrical properties of the chamber and in particular the stored electrical energy and the requirements to protect the chamber from this energy. Section IV discusses the solution chosen to protect the chamber including the choice of high voltage (HV) switch. Section V

Manuscript received October 29, 2003. This work was supported in part by the U.S. Department of Commerce under Grant No. BS123456 (sponsor acknowledgment goes here).

M. Binkley is with Fermi National Accelerator Laboratory, Batavia, IL 60510, USA (telephone: 630-840-3112, e-mail: binkley@fnal.gov).

A. Mukherjee is with Fermi National Accelerator Laboratory, Batavia, IL 60510, USA (telephone: 630-840-2390, e-mail: mukherjee@fnal.gov).

W. Stuermer is with Fermi National Accelerator Laboratory, Batavia, IL 60510, USA (telephone: 630-840-4187, e-mail: stuermer@fnal.gov).

R. L. Wagner is with Fermi National Accelerator Laboratory, Batavia, IL 60510, USA (telephone: 630-840-8115, e-mail: wagner@fnal.gov). describes the final product installed in the experiment. Section VI gives the conclusions.

\section{Mechanical Properties of the COT}

The COT measures about $3 \mathrm{~m}$ long by $3 \mathrm{~m}$ in diameter. It resides inside a $1.4 \mathrm{~T}$ solenoid magnet in the middle of the CDF detector. The cylindrical chamber has more than 30,000 sense wires and 40,000 field shaping (potential) wires arranged radially into 8 annular regions called super-layers (4 super-layers are axial and 4 are small angle stereo). Inside a super-layer the wires are organized in a jet cell configuration as shown in Fig. 1. All the wires are at high voltage.

There are 12 sense wires layers (voltages) per cell giving a total of 96 sense wire layers in the 8 super-layers. Each wire layer corresponds to a specific radius in the COT. There are potential wires between each pair of sense wires and at each end of a cell. The three potential wires at each end of the cell are tied to the same voltage which means that 13 potential wire voltages are required per cell. Therefore a cell requires 25 voltages and all the cells in a super-layer are run with the same 25 voltages. The eight super-layers can be operated independently; however, the relative voltages of the 25 high voltages supplies (wire layers) for a superlayer have definite

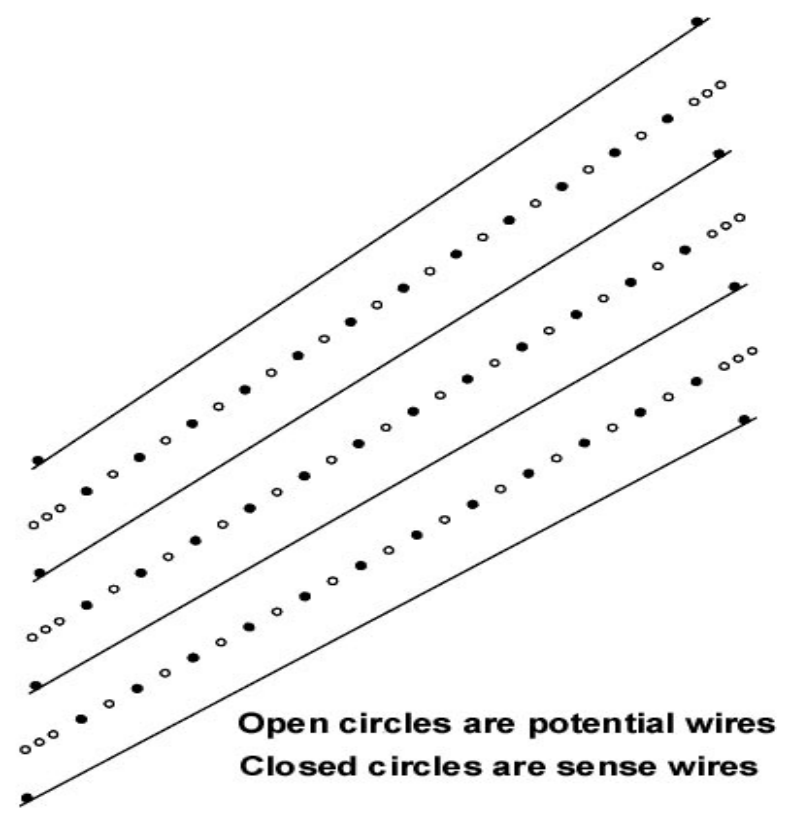

Fig. 1. Wire layout for three cells in the COT. 


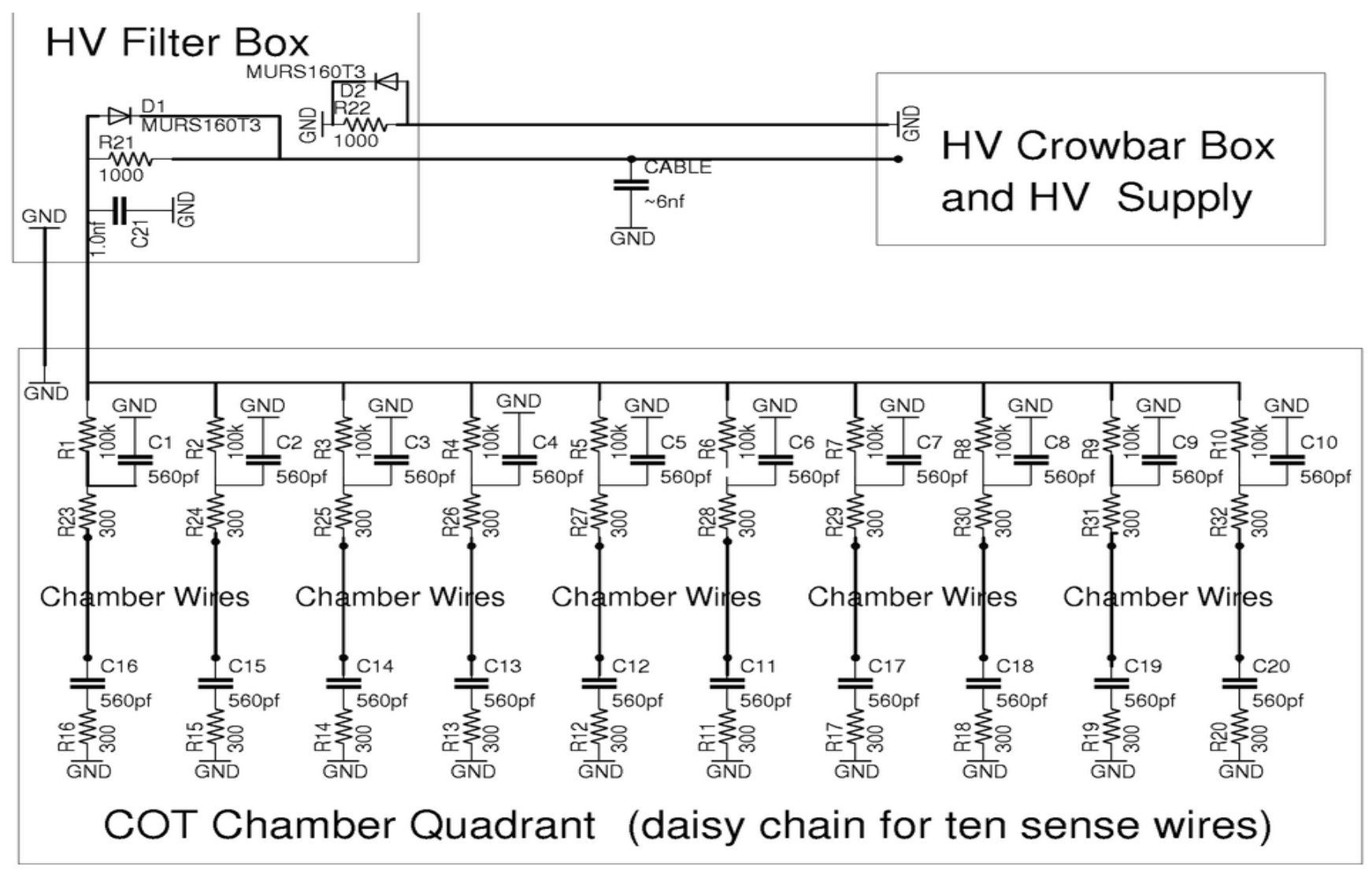

Fig. 2. A simplified schematic of the HV distribution for a single sense wire layer showing only ten wires.

limits in order to prevent breakdown between adjacent wires.

All the wires are $40 \mu \mathrm{m}$ gold-plated tungsten. The maximum drift distance is about $1 \mathrm{~cm}$ and the drift gas is a $50 / 50 \mathrm{mix}$ of argon/ethane with an admixture of $1.7 \%$ isopropanol to reduce chamber aging. The cathode planes are $35 \mathrm{~nm}$ gold on a thin mylar film.

\section{High Voltage Electrical Properties of the COT}

Since there are 25 sense and potential wire voltages (layers) per super-layer, a total of $200 \mathrm{HV}$ supplies are required for all 8 super-layers. All the wires in their radial layer are connected together using four daisy chains (one per quadrant) before being attached to one of the $200 \mathrm{HV}$ supplies.

Fig. 2 shows a simplified schematic for the connection of one sense wire layer to its high voltage supply (including a daisy chain for only ten wires). Each sense wire has two $560 \mathrm{pF}$ capacitors. One is a filter capacitor for a $300 \Omega$ terminating resistor and the other is a blocking capacitor for the preamplifier at the far end of the wire (represented in the schematic by its $300 \Omega$ input impedance). A potential wire layer is the same except that the current limiting resistors R1$\mathrm{R} 10$ are $200 \mathrm{k} \Omega$ and the preamplifiers and terminating resistors (R11-R20 and R23-R32) are replaced by jumpers. Each sense or potential wire layer has a wire in every cell in its superlayer. At the nominal operating voltage of $3 \mathrm{kV}$ for the sense wires, the value of the two $560 \mathrm{pF}$ capacitors in parallel degrades from $1,120 \mathrm{pF}$ to about $800 \mathrm{pF}$. Table I gives the properties of a sense wire layer as a function of superlayer. The number of cells is the same as the number of wires in a layer. "Rsense" and "Csense" are the total parallel resistance and capacitance on the chamber. The stored energy per layer (E/Layer) for a sense wire can be as much as $2.2 \mathrm{~J}$ which includes the energy in the high voltage filter box and the cables. The potential wire voltage is little more than half of the nominal sense wire voltage giving appreciably smaller stored energies.

The problem is to quickly dump the energy stored in the high voltage daisy-chains and cables when an over-current condition is sensed in the chamber. If the over-current condition is caused by a discharge in the chamber, this

Table I. Electrical properties of the COT sense wires.

\begin{tabular}{|l|l|l|l|l|l|l|}
\hline SL & Cells & $\begin{array}{l}\text { Rsense } \\
(\Omega)\end{array}$ & $\begin{array}{l}\text { Csense } \\
(\mu \mathrm{F})\end{array}$ & $\begin{array}{l}\text { E/Layer } \\
(\mathrm{J})\end{array}$ & $\begin{array}{l}\text { Rcb } \\
(\Omega)\end{array}$ & $\begin{array}{l}\tau \\
(\mu \mathrm{s})\end{array}$ \\
\hline 1 & 168 & 595 & 0.134 & 0.8 & 1000 & 214 \\
\hline 2 & 192 & 521 & 0.154 & 0.9 & 1000 & 234 \\
\hline 3 & 240 & 417 & 0.192 & 1.1 & 820 & 238 \\
\hline 4 & 288 & 347 & 0.230 & 1.3 & 820 & 268 \\
\hline 5 & 336 & 298 & 0.269 & 1.5 & 820 & 301 \\
\hline 6 & 384 & 260 & 0.307 & 1.7 & 820 & 332 \\
\hline 7 & 432 & 231 & 0.346 & 1.9 & 750 & 339 \\
\hline 8 & 480 & 208 & 0.384 & 2.2 & 750 & 368 \\
\hline
\end{tabular}




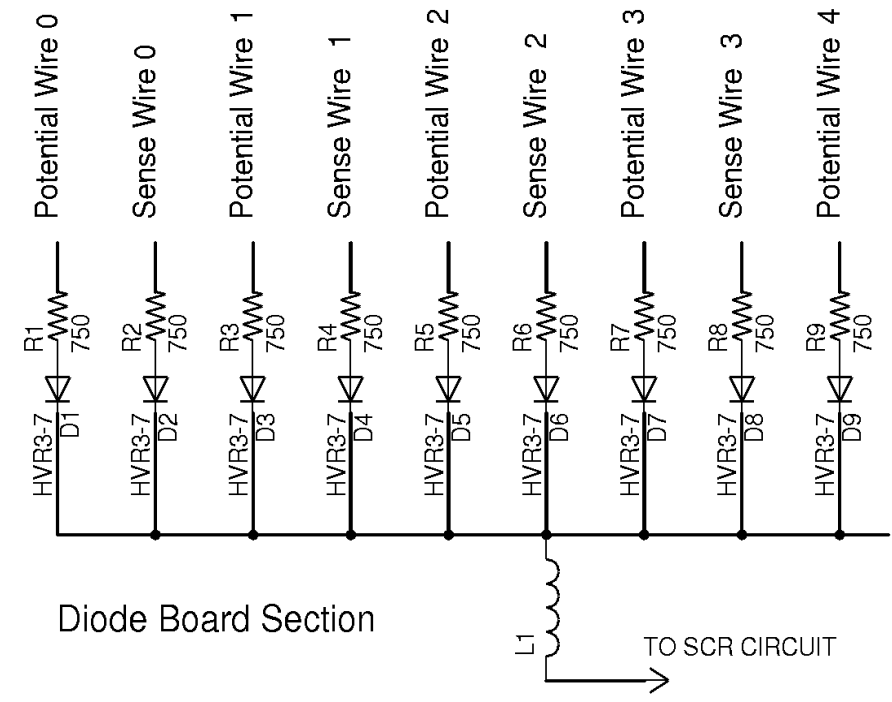

Fig. 3. Section of Diode Board that fans in the voltages of the sense and potential wires (only 9 of 25 shown).

stored energy will recharge the problem wire with a time constant of about $80 \mu \mathrm{s}$ which sets the time scale of interest. Dumping the energy with a mechanical switch is too slow (of order $1 \mathrm{~ms}$ ). A solid state high voltage switch is needed!

\section{The High Voltage Switch}

The energy on all the wires in a superlayer must be dumped simultaneously. For each of the 25 high voltage layers in a superlayer, the cables from the four quadrants and the $\mathrm{HV}$ supply must be bussed together. A good solution is to fan in these 25 busses through high voltage diodes to a common high voltage switching device. The general fan-in for a partial super-layer is shown in Fig. 3. The resistors limit the current

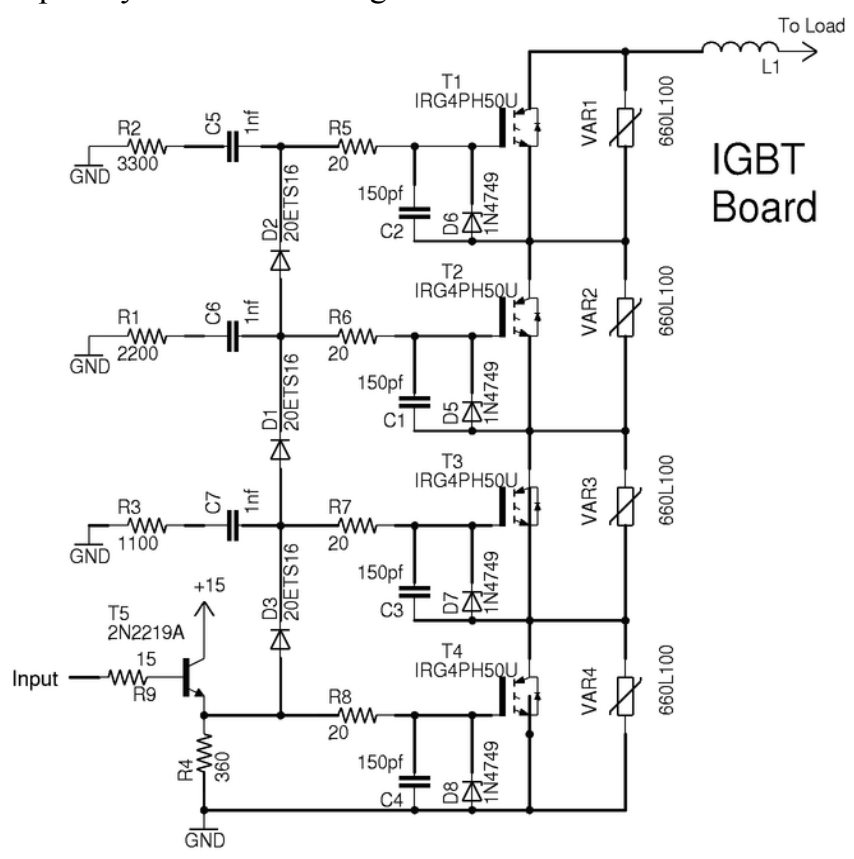

Fig. 4. Schematic for the IGBT crowbar option. so that the peak current for a super-layer is of order $100 \mathrm{~A}$ at full voltage. All potential wire resistors are $750 \Omega$; the value of the sense wire resistors Rcb varies to reduce the differences in discharge time constant $\tau$ (see Table I).

The inductor shown in Fig. 3 as part of the diode board is inserted to protect the switching device by limiting the size of the discharge current until the device is fully turned on.

Finding an inexpensive commercial HV inductor was difficult, so they were hand made at Fermilab. They consist of about 40 turns of teflon insulated solid 26 gauge copper wire on a wooden bobbin $2.86 \mathrm{~cm}$ in diameter by $4.45 \mathrm{~cm}$ long. The inductor is visible in Fig. 8 which shows the inside of a crowbar box. Inside the wooden bobbin there is a $4.19 \mathrm{~cm}$ long ferrite bead (\#43 by Fair-Rite Products). With the ferrite bead the inductance is about $100 \mu \mathrm{H}$ giving an $\mathrm{L} / \mathrm{R}$ of order $2 \mu \mathrm{s}$.

Initially a high voltage switching circuit was developed that used off-the-shelf 1,200 V insulated gate bipolar transistors (IGBTs) manufactured by International Rectifier. The circuit shown in Fig. 4 uses a stack of four IGBTs with Harris LA series varistors limiting the voltage across each IGBT. A positive input pulse turns on T4, the lowest voltage IGBT and the dropping of its collector voltage turns on T3, the IGBT above it. In turn, the dropping of the collector voltage on each IGBT turns on the next IGBT in the stack until all are conducting.

The problem with the IGBT circuit appeared to be getting the IGBTs to turn on uniformly. The IGBTs are very fast and tend to ring. Adding damping capacitors $\mathrm{C} 1-\mathrm{C} 4$ and being very careful to keep the layout compact helped. One such prototype was made that successfully discharged a $13 \mu \mathrm{F}$ capacitor at $3,500 \mathrm{~V}$ through $35 \Omega$ every $5 \mathrm{~min}$ for several days. However there was concern that this circuit was too

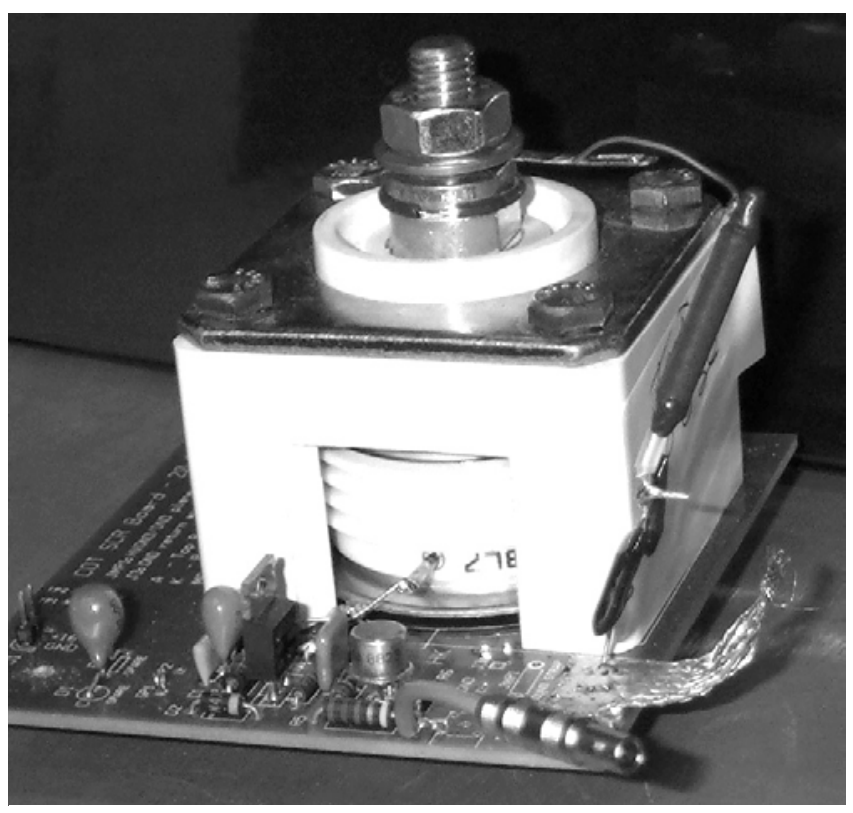

Fig. 5. The hockey puck T201N Eupec SCR used in the final design shown in its ceramic housing. 


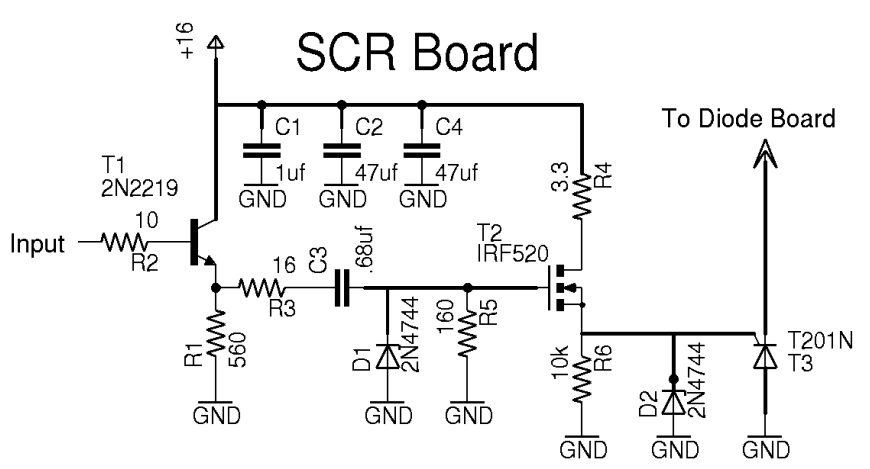

Fig. 6. Schematic for the circuitry to fire the SCR.

sensitive to layout considerations and another solution was sought. A single high voltage silicon-controlled rectifier (SCR) was found that was easier to work with. The choice was the hockey puck style T201N SCR made by Eupec. It is about $5.8 \mathrm{~cm}$ in diameter and $2.54 \mathrm{~cm}$ high and mounted in a ceramic housing. It is shown mounted in its housing in Fig. 5. The $\mathrm{T} 201 \mathrm{~N}$ has a reverse voltage rating of $7 \mathrm{kV}$. Engineers from Eupec recommended using a device rated for twice the normal operating voltage in our application since the duty factor was expected to be close to $100 \%$. A device operating close to its rating for a long period of time has a small but finite chance of damage due to a cosmic ray interaction. The $\mathrm{T} 201 \mathrm{~N}$ is rated for an average on-state current of $200 \mathrm{~A}$ and a surge current of 2,500 A, both well above the peak current in our application. More important is the critical rate of current rise of $150 \mathrm{~A} / \mu$ s with a gate current of $3 \mathrm{~A}$. We quickly destroyed our initial SCR by running with a large rate of current rise (i.e. without a protection inductor) and at a gate current of less that $1.0 \mathrm{~A}$.

Fig. 6 shows the circuit for firing the SCR. The input is a positive $16 \mathrm{~V}$ pulse that is generated by a pnp transistor switch (2N3906) when the crowbar box input goes low. This signal passes through an emitter follower to turn on the IRF520 mosfet. The T201N gate sees an initial current pulse of $3.5 \mathrm{~A}$ through the $3.3 \Omega$ resistor $\mathrm{R} 4$. The scope picture in Fig. 7 shows a crowbar box discharging through its diode board a $13 \mu \mathrm{F}$ capacitor charged to $3 \mathrm{kV}$. Trace 1 (at the bottom) shows the TTL level input pulse that initiates the discharge by going to the low voltage state. Trace 2 (starting nearest the top) shows the voltage on the drain of the IRF520 giving a measure to the voltage across the $3.3 \Omega$ resistor and thereby the SCR gate current. Traces 3 and 4 (both starting at marker 4) are the voltages after and before the protection inductor using a high voltage capacitive divider probe $(3 \mathrm{pF}$ to $560 \mathrm{pF}$ with a $5.6 \mathrm{~ms} \mathrm{RC}$ ). The math trace (starting at marker $\mathrm{M}$ near the bottom) is the difference between traces 3 and 4 showing that the voltage across the inductor returns to zero after a few $\mu$ s. During burn in, each crowbar box underwent at least 1,000 such discharge cycles. Since adding the protection inductor and increasing the SCR gate current, there have been no SCR failures either in testing or during operations.

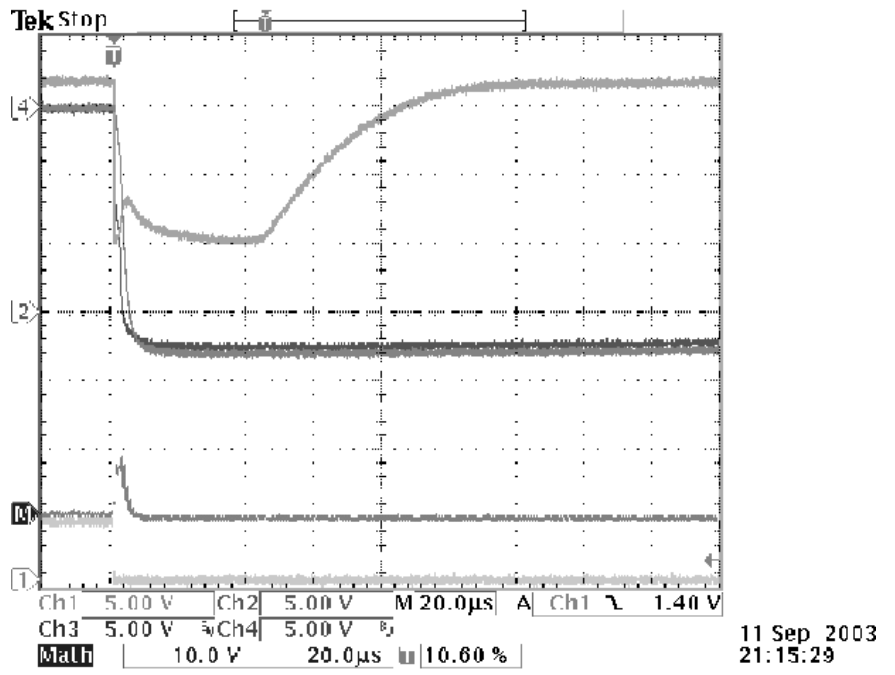

Fig. 7. Scope trace of a test SCR discharge on a time scale of $20 \mu \mathrm{s} / \mathrm{cm}$.

\section{The Packaged PRoduct.}

The crowbar box circuitry for one super-layer is built into a standard $48.3 \mathrm{~cm} \times 13.3 \mathrm{~cm} \times 35.6 \mathrm{~cm}$ rack mounted chassis. Fig. 8 shows the inside of a crowbar box. On the back panel there are 25 rows of SHV connectors, each row corresponding to one of the 13 potential wire or 12 sense wire layers in each super-layer. Each row has 5 connectors that are bussed together: the top connector goes to the HV supply and the other four go to the four quadrants of the detector. There are 25 white jumpers from the bussed rear panel rows to the 25 channels on the diode board (Fig. 3). After going through a

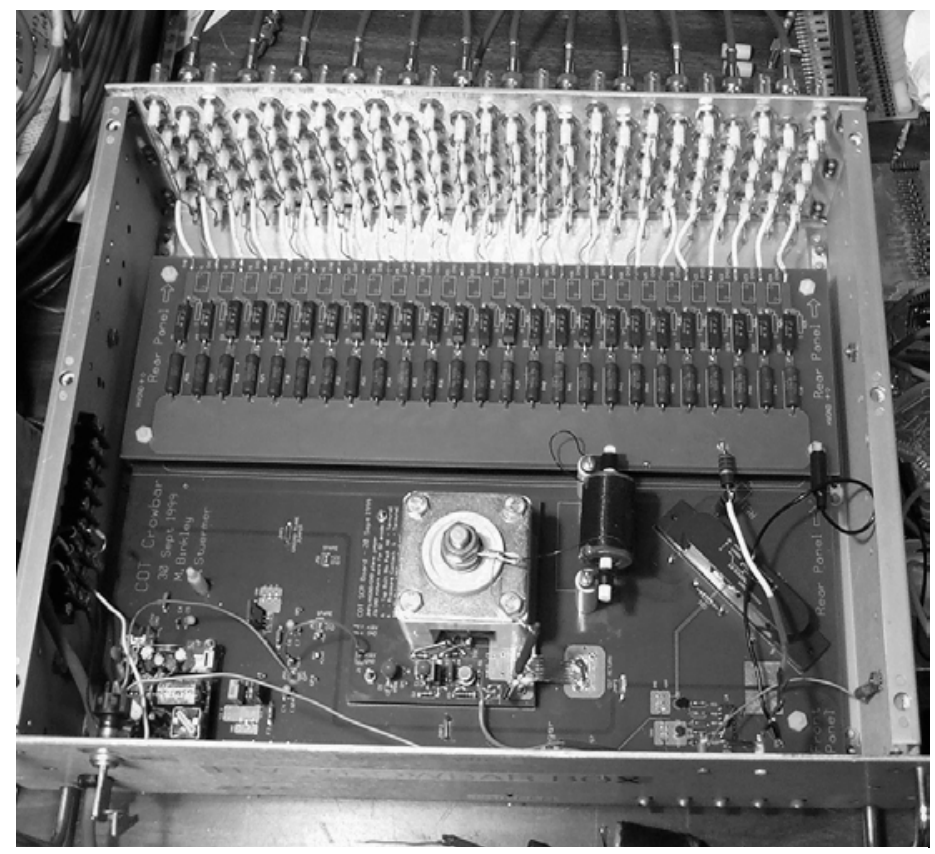

Fig. 8. Inside of a crowbar box showing the back panel, diode board, inductor, and SCR. 
HV diode and resistor, all 25 channels are bussed together and connected through the protection inductor (black coil) to the SCR drain (stud with large nut on top of the ceramic housing at the middle front of the box). The diode board bus is also connected to a mechanical high voltage relay (Magnecraft W102VX-51) just to the right of the inductor which holds the bus to ground for extended periods of time. There also is a $\mathrm{HV}$ monitor output through 2,000 M $\Omega$ to test points on the front panel (upper right). The SCR drive circuitry (Fig. 6) is on a separate board under the SCR (middle front of the box) allowing for quick replacement. At the left front of the box is a Condor GSM7-24 power supply ( $24 \mathrm{~V}$ at $7 \mathrm{~W}$ ) that powers all the low voltage electronics. At the upper left hand corner of the front panel is a key that can lock the crowbar box in a tripped state. At the lower right hand corner of the front panel are 5 lemo TTL inputs, any one of which puts the box in a tripped state when it goes low. Each crowbar box services one super-layer.

\section{CONCLUSIONS}

Eight crowbar boxes, one for each superlayer in the COT, have been in service for three years. They use a HV SCR switch that reliably discharges all the stored energy in a superlayer when an over-current condition is sensed. Before installation in the experiment, each crowbar box underwent rigorous testing which included discharging a $13 \mu \mathrm{F}$ capacitor charged to $3.5 \mathrm{kV}$ more than 1,000 times. In the experiment, each crowbar box has discharged its superlayer thousands of times, hundreds of times at full voltage. There have been no failures to date.

\section{ACKNOWLEDGMENTS}

The authors wish to thank the Fermilab staff for their vital contributions. This work was supported by the U.S. Department of Energy.

\section{REFERENCES}

[1] R. Blair, K. Byrum, J. Dawson, V. Guarino, S. Kuhlmann, T. LeCompte, et al., "The CDF II Detector: Technical Design Report," Fermilab-Pub96-390-E (1996).

[2] J. T. Affolder, D. Allspach, D. Ambrose, J. Bialek, W. Bokhari, M. Brozovic, et al., "CDF Central Outer Tracker," Fermilab-Pub-03/355-E (2003), to be published in Nucl. Instr. and Meth. 